Hans-Joachim Alscher*

\title{
Die Niederösterreichische Landesbibliothek
}

DOI 10.1515/bfp-2016-0006

Zusammenfassung: Der Autor porträtiert die Niederösterreichische Landesbibliothek und zeichnet ihren Werdegang von der Gründung im Jahre 1813 in Wien, der früheren Hauptstadt von Niederösterreich, bis zur Übersiedlung in die neue Landeshauptstadt Sankt Pölten im Jahre 1997 nach und berichtet über Veränderungen, die bis zur Gegenwart reichen. Seit 1982 ist die Bibliothek mit dem Pflichtexemplarrecht ausgestattet.

Schlüsselwörter: Niederösterreich; Wissenschaftliche Bibliothek; Landesbibliothek; Sankt Pölten

\section{State Library of Lower Austria}

Abstract: The author portrays the State Library of Lower Austria, its history from the very beginnings in 1813 at Vienna, the former capital town of Lower Austria, and informs about relocation to the new capital town Sankt Pölten in 1997, and current changes in operation schedule. Since 1982, the status of a depository library has been guaranteed by Austrian federal law.

Keywords: Lower Austria; scientific library; state library; Sankt Pölten

\section{Inhalt}

1 Wer wir sind, wofür wir stehen: die synchrone

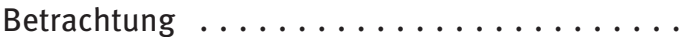

2 Wie es dazu kam, was vorher war: die diachrone Betrachtung ................

\section{Wer wir sind, wofür wir stehen: die synchrone Betrachtung}

Als derzeitiger Bibliotheksdirektor komme ich gerne der Einladung nach, dieses Haus einem fachkundigen Publikum vorzustellen. „Landesbibliothek“ - das ist die wissenschaftliche Bibliothek eines Landes, mit Aufgaben ähnlich

*Kontaktperson: wHR Mag. Hans-Joachim Alscher, hans.alscher@noel.gv.at denen einer Nationalbibliothek, nur eben auf den kleineren Raum eines Bundeslandes beschränkt. Damit gehen auch Rechte einher, insbesondere das Recht auf Bibliotheksstücke gemäß §§ 43-45 Mediengesetz in Verbindung mit der Pflichtablieferungsverordnung, die im Wesentlichen am 1.1.1982 in Kraft getreten ist. ${ }^{1} \mathrm{Zu}$ den bibliografischen Bestrebungen zählt die laufende Erstellung einer thematischen Auswahlbibliografie zu Niederösterreich, die früher einmal jährlich als gedruckte Beilage zu der vom Verein für Landeskunde von Niederösterreich herausgegebenen Quartalszeitschrift „Unsere Heimat“ erschien, später online in PDF-Format zur Verfügung gestellt wurde und inzwischen als vorgefertigte Datenbankabfrage zum Onlinekatalog angeboten wird. Künftig soll eine Regionalbibliografie von in Niederösterreich erschienenen Ressourcen in gleicher Weise zugänglich sein. Als Landesbibliothek sehen wir unsere wichtigste Aufgabe darin, das gesamte Schrifttum aus Niederösterreich, von niederösterreichischen Autoren oder zu niederösterreichischen Themen zu sammeln, zu erschließen und dauerhaft verfügbar zu halten.

Vom vertraglich vereinbarten Sonderfall der Universitäts- und Landesbibliothek Tirol abgesehen, ist das in Deutschland hauptsächlich verbreitete Konzept einer Universitäts- und Landesbibliothek in Österreich nicht üblich. Der Hintergrund ist, dass das Universitätswesen in Österreich (im Unterschied zu Deutschland) Bundessache, eine Landesbibliothek hingegen eine Einrichtung des jeweiligen Landes ist. Von der Nationalbibliothek als Nachfolgerin der Kaiserlich-Königlichen Hofbibliothek und gesamtstaatlichen Einrichtung abgesehen, existiert ein gewisser Dualismus zwischen einer Landesbibliothek und der für das Gebiet dieses Landes zuständigen Universitätsbibliothek. Auf Erzherzogin Maria Theresia geht die Idee zurück, dass in jedem Kronland eine Universitätsbibliothek oder

1 Diesen Rechtsvorschriften des Bundes zufolge hat von jedem Druckwerk, das in Niederösterreich verlegt wird oder erscheint, der Medieninhaber (Verleger), wenn das Druckwerk aber im Ausland verlegt wird und erscheint, jedoch in Niederösterreich hergestellt wird, der Hersteller binnen eines Monats nach Beginn der Verbreitung bzw. nach Herstellung an die Österreichische Nationalbibliothek zwei Stücke, an die Universitätsbibliothek Wien ein bzw. zwei Stücke und an die Niederösterreichische Landesbibliothek zwei bzw. drei Stücke auf eigene Kosten abzuliefern (die höhere Anzahl gilt im Falle periodischer, das heißt mindestens viermal im Kalenderjahr erscheinender Druckwerke). 
ersatzweise eine eigens eingerichtete staatliche Studienbibliothek die Aufgabe der öffentlichen Literaturversorgung $^{2}$ übernimmt. Letztlich entspricht dieser föderale Dualismus somit dem Spannungsverhältnis Landesfürst und dessen Statthalter (später Bundesverwaltung) gegen Stände (später Landesverwaltung). Als letzte der zuvor erwähnten Studienbibliotheken wurde die Bundesstaatliche Studienbibliothek Linz, die mit der (später entstandenen) Universitätsbibliothek Linz konkurrierte, vom Land Oberösterreich übernommen und zur Oberösterreichischen Landesbibliothek umfunktioniert. Aus dem zuvor genannten Grund waren die Universitätsbibliotheken früher direkt dem Bundesministerium für Wissenschaft und Forschung unterstellt und wurden erst mit dem Universitäts-Organisationsgesetz $1993 \mathrm{zu}$ unselbstständigen Einrichtungen der jeweiligen Universität degradiert. In der ursprünglichen Aufgabenstellung der öffentlichen Literaturversorgung, die mit der neuen Abhängigkeit zumindest in Frage gestellt ist, liegt aber das Pflichtexemplar-Recht der Universitätsbibliotheken (parallel zu dem der meist später entstandenen Landesbibliotheken) begründet. Dass für Niederösterreich die Universitätsbibliothek Wien zuständig ist, hat natürlich den historischen Grund, dass Wien die frühere Hauptstadt Niederösterreichs war. Die Donau-Universität Krems existierte hingegen 1982 noch nicht, sodass eine Universität auf niederösterreichischem Boden als Alternative bei Inkrafttreten des Mediengesetzes nicht vorhanden war. Mangels Existenz einer eigenen Universität im Burgenland ist die Universitätsbibliothek Wien neben Wien und Niederösterreich auch für das Burgenland zuständig.

Die Funktion der (öffentlich zugänglichen) Landesbibliothek ist nicht unsere einzige: Wir sind des Weiteren Amtsbibliothek der NÖ Landesregierung, eine Behördenbibliothek mit der Aufgabe, die internen Informationsbedürfnisse der Dienststellen des Landes abzudecken, wozu neben Recht auch Themenbereiche wie Politik und Verwaltung, Wirtschaft und Gesellschaft zählen. Das ist deswegen der Rede wert, weil es in einigen anderen Bundesländern eine Trennung dieser Funktionen gibt, sodass eine Amtsbibliothek der Landesregierung neben der Landesbibliothek existiert. Eine weitere Besonderheit ist, dass wir seit 1886 auch als Bibliothek des 1864 gegründeten Vereines für Landeskunde von Niederösterreich fungieren, dessen eigene Bibliothek damals eingegliedert wurde, was sich in Sammelschwerpunkten wie Geschichte, Kunstgeschichte, Landeskunde, Heimatkunde und Volkskunde

2 Nicht nur der Lehrenden und Studierenden der jeweiligen Institution. niederschlägt. Über diese Kernbereiche hinaus erwerben wir im Rahmen unserer Möglichkeiten Basisliteratur zu allen anderen Wissensgebieten und verstehen uns als wissenschaftliche Allgemeinbibliothek. Ein besonderes Augenmerk gilt der Belletristik sowie der Kinder- und Jugendliteratur, soweit es sich nicht um Klassiker der Weltliteratur oder um niederösterreichrelevante Ressourcen im Sinne unserer Funktion als Landesbibliothek handelt.

Wir verstehen uns als Serviceeinrichtung, zu deren Besonderheiten es gehört, dass wir nicht nur unentgeltliche Entlehnung (wir sind keine reine Präsenzbibliothek) und die üblichen Leistungen der nationalen wie internationalen Fernleihe einschließlich document delivery anbieten, sondern darüber hinaus Medien an registrierte Leser unentgeltlich mit der Post zusenden (Versandleihe). Da der gesamte Bibliotheksbestand natürlich online recherchierbar ist und sowohl die Registrierung als auch die Bestellung online erfolgen kann, besteht für die Zwecke der Entlehnung keine unmittelbare Notwendigkeit, die Räumlichkeiten der Bibliothek aufzusuchen. ${ }^{3}$ Die Notwendigkeit des persönlichen Erscheinens besteht vor allem für die Benutzung nicht entlehnbarer oder nicht leicht reproduzierbarer Ressourcen, insbesondere für die Inanspruchnahme der Freihandbestände im Lesesaal. Von der Entlehnung ausgenommen sind neben verschiedenen Sondermedien (topografische Ansichten, Landkarten, weitere Non-Book-Materialien, Zeitungen und Zeitschriften) Druckschriften, deren Wert oder Zustand dies nicht erlauben: alle, die vor 1900 erschienen sind. Für Bürger des Waldviertels (nordwestliches Niederösterreich) gibt es die Möglichkeit, solche Medien zu bestimmten Zeiten in den Räumlichkeiten der Bezirkshauptmannschaft Waidhofen an der Thaya zu benutzen. Ein Wunsch besteht darin, eine solche Möglichkeit an allen (derzeit) 21 Bezirkshauptmannschaften zu schaffen, um die Dienstleistungen der Bibliothek den Nutzern im räumlichen Sinne näherzubringen.

Jede Bibliothek hat ihre historisch gewachsenen Besonderheiten: Zu unseren Stärken zählt die Existenz einer bedeutenden Sammlung topografischer Ansichten sowie einer Kartensammlung, ${ }^{4} \mathrm{zu}$ unseren Schwächen das fast völlige Fehlen von Handschriften. Letzteres erklärt sich daraus, dass wir trotz unseres Alters zu jung sind, als dass wir von den in Österreich hauptsächlich unter Kaiser Jo-

3 Die Organisation und Art der Rückgabe der entlehnten Medien obliegt dem Benutzer.

4 Die Bestände des 1984 als Burgenkundliche Sammlung eingegliederten NÖ Burgenarchivs von Prof. Felix Halmer wurden 2001 nach der Pensionierung des Kurators Hon.-Prof. Dr. Hermann Steininger auf die anderen Sammlungen aufgeteilt. 
seph II. durchgeführten Klosteraufhebungen in den Jahren 1780 bis 1790 hätten profitieren können. Wir schmücken uns auch nicht mit Beutegut (der Reichsdeputationshauptschluss von 1803 spielte in Niederösterreich hingegen keine nennenswerte Rolle).

Neben der Homepage ${ }^{5}$ besteht die Visitenkarte einer Bibliothek aus ihrem Katalog, genauer aus dem OPAC (Online Public Access Catalogue) ${ }^{6}$ als Publikumsschnittstelle. In unserem Fall liegt dem Katalog das seit 1994 im Einsatz befindliche Bibliothekssystem BIS-C 2000 der DABIS GmbH - Gesellschaft für Datenbank-Informationssysteme $^{7}$ in Wien zugrunde, welches eine eigenständige Datenbank bildet, somit nicht als bloße Oberfläche auf einer Standarddatenbank wie zum Beispiel ORACLE aufsetzt. $\mathrm{Zu}$ den verwendeten Standards zählt die Gemeinsame Normdatei (GND), die wöchentlich von der Deutschen Nationalbibliothek im Format MARC 21 XML bezogen, in ein spezifisches Lokalformat konvertiert und in die Katalogdatenbank importiert wird, folglich in aufbereiteter Form fester Bestandteil des Kataloges ist. Die Sacherschließung erfolgt in Form einer Schlagwortkatalogisierung nach den Regeln für den Schlagwortkatalog (RSWK) in Verbindung mit den GND-Übergangsregeln. Die bisher nach den auf der International Standard Bibliographic Description (ISBD) beruhenden Regeln für die alphabetische Katalogisierung in wissenschaftlichen Bibliotheken (RAK-WB) vorgenommene Formalerschließung wird derzeit auf das neue, auf den Functional Requirements for Bibliographic Records (FRBR) und Functional Requirements for Authority Data (FRAD) basierende Regelwerk Resource Description and Access (RDA) umgestellt.

Im Zuge des Wechsels von den früheren Normdateien Personennamendatei (PND), Gemeinsame Körperschaftsdatei (GKD) und Schlagwortnormdatei (SWD) auf die Gemeinsame Normdatei (GND) wurden 2012 sämtliche BIS-CDatenbanken der Landesbibliothek sowie weiterer Landeseinrichtungen zu einer einzigen Datenbank zusammengeführt, sodass der Katalog der Niederösterreichischen Landesbibliothek nicht nur wie vordem den Bestand der Druckschriftensammlung inklusive deren Altbestände, sondern auch die Bestände der Kartensammlung, der Topografischen Sammlung, der in der Landesbibliothek verwalteten Vor- und Nachlässe der Literaturdokumentation Niederösterreich, ${ }^{8}$ der Plakate und darüber hinaus die Bestände der Archivbibliothek (Bibliothek des Niederöster-

5 Siehe www.noe.gv.at/landesbibliothek bzw. Umleitung von www. noelb.at.

6 Siehe bibliothekskatalog.noe.gv.at.

7 Siehe www.dabis.eu.

8 Z. B. von Karl Farkas oder von Carl Merz. reichischen Landesarchivs und des NÖ Instituts für Landeskunde), die bibliografischen Datensätze der seinerzeit vom NÖ Institut für Landeskunde betreuten Niederösterreichischen Bibliographie - Artikel und Aufsätze in Zeitschriften und Periodika, die Bestände der Gruppe Wasser sowie der Abteilung Kinder- und Jugendhilfe des Amtes der Niederösterreichischen Landesregierung und des Archäologischen Parks Carnuntum beinhaltet. Natürlich ist eine Einschränkung der Suche auf einen oder mehrere dieser Teilbestände möglich (vorausgewählt ist die Druckschriftensammlung). Diese nunmehr integrierten Datenbanken haben zum Teil nicht den gleichen Qualitätsstandard wie die Druckschriftensammlung aufgewiesen. An der Verbesserung dieser Daten wird noch gearbeitet. Gerne wird die vor allem beim Bestand der Topografischen Sammlung vorzufindende Einbettung digitaler Abbildungen (Thumbnails) der Sammlungsobjekte im jeweiligen Katalogisat angenommen. Neu hingegen ist die Möglichkeit, solche Datensätze in Social Media wie Facebook zu „teilen“, die Bestände der Topografischen Sammlung sind im „Kulturpool“ ${ }^{“ 9} \mathrm{zu}$ finden. Neben dem für das Projekt ALEX, Historische Rechts- und Gesetzestexte Online, ${ }^{10}$ zur Verfügung gestellten Landesgesetzblatt für Niederösterreich (1849-1971) ${ }^{11}$ werden die wesentlichen Publikationen des Vereines für Landeskunde von Niederösterreich ${ }^{12}$ für die Zeit von 1865 bis 1999 online als PDF-Dateien angeboten. Über die Homepage besteht Zugriff auf die Onlineausgaben unserer Eigenpublikationen, vor allem der Kataloge der „Sonder- und Wechselausstellungen der Niederösterreichischen Landesbibliothek“, die im Vergleich zur Druckausgabe mit Abbildungen aller ausgestellten Objekte angereichert sind.

Der Bibliothekskatalog bildet keinen Bestandteil des sogenannten Österreichischen Bibliothekenverbundes, der von der Österreichischen Bibliothekenverbund und Service GmbH (obvsg) betrieben wird und auf dem ORACLE-basierten System Aleph 500 bzw. dessen Nachfolger Alma (Ex Libris Group) aufsetzt. Es handelt sich bei diesem Verbund um den Nachfolger des BIBOS-2-Verbundes, der die dem Bundesministerium für Wissenschaft und Forschung unterstellten Universitätsbibliotheken sowie die

9 Siehe www.kulturpool.at.

10 Siehe alex.onb.ac.at, abgespalten von ANNO - AustriaN Newspapers Online, Historische österreichische Zeitungen und Zeitschriften online (anno.onb.ac.at).

11 Ohne die Loseblattausgabe 1972-2014 bzw. die authentische Kundmachung im Rechtsinformationssystem des Bundes (RIS) ab 2015.

12 Jahrbuch für Landeskunde von Niederösterreich, Zeitschrift Unsere Heimat, jeweils samt Vorläufern. 
Nationalbibliothek umfasste. Altbestände einzelner Verbundbibliotheken sind auch heute noch lediglich als Card Image Public Access Catalogue (CIPAC) auf Grundlage von KatZoom verfügbar. Ein überregionaler Zugang zu unserem Katalog ist allerdings über den Karlsruher Virtuellen Katalog $(\mathrm{KVK})^{13}$ gegeben, der ein Suchportal zu vielen Bibliotheks- und Verbundkatalogen vor allem des deutschen Sprachraumes darstellt. Eine aktuelle Kopie unseres Bibliothekskataloges bildet einen Bestandteil des dort zur Verfügung stehenden, BIS-C-basierten, virtuellen Verbundes der Landesbibliotheken Österreich und Südtirol. ${ }^{14}$ Diesem Verbund entspricht auch der Österreichische Normdatenverbund (ÖNDV) als eigenständige Redaktion ${ }^{15}$ der Gemeinsamen Normdatei (GND), der federführend von der Niederösterreichischen Landesbibliothek ${ }^{16}$ betreut wird.

Organisatorisch bildet die Niederösterreichische Landesbibliothek seit Mai 2011 eine Referatsgruppe der Abteilung NÖ Landesarchiv und NÖ Landesbibliothek des Amtes der NÖ Landesregierung (K2) unter der Leitung von Archivdirektor Willibald Rosner innerhalb der Gruppe Kultur, Wissenschaft und Unterricht des Amtes der NÖ Landesregierung (K) unter der Leitung von Joachim Rössl. ${ }^{17}$ Politisch zuständiges Mitglied der NÖ Landesregierung ist Landeshauptmann-Stellvertreter Mag. Wolfgang Sobotka (seit 2005). ${ }^{18}$ Vor dem Mai 2011 bildeten die Abteilung NÖ Landesarchiv und NÖ Institut für Landeskunde (K2) unter der Leitung von Archivdirektor Willibald Rosner und die Abteilung NÖ Landesbibliothek (K3) unter der Leitung von Bibliotheksdirektor Gebhard König getrennte Organisationseinheiten. Standort der Bibliothek ist seit 1997 das Haus Kulturbezirk $3^{19}$ in der Landeshauptstadt Sankt Pölten. Ein gemeinsam mit dem NÖ Landesarchiv und dem NÖ Landesmuseum betriebener Nebenstandort ist das „Kulturdepot“, ein 2009 fertiggestelltes Magazingebäude am westlichen Stadtrand. Rechtsgrundlage der Landesbibliothek ist die von der NÖ Landesregierung beschlossene Bibliotheksordnung von 1997 und die darauf basierende, vom Bibliotheksdirektor erlassene Benutzungsordnung, darüber hinaus die Verordnungen über die Geschäftsordnung der NÖ Landesregierung bzw. des Amtes der NÖ Landesregierung und indirekt die Pflichtablieferungsverordnung zum Mediengesetz. Die Ausbildung ist durch die Verordnungen der NÖ Landes-

13 Siehe www.ubka.uni-karlsruhe.de/kvk.html.

14 Siehe www.landesbibliotheken.eu.

15 Kennungen 1402 und 1403.

16 ISIL: AT-NOELB.

17 Ab 1.1.2016: Hermann Dikowitsch.

18 Zuvor: Landeshauptmann-Stellvertreterin Liese Prokop.

19 Siehe www.kulturbezirk.at. regierung über die Prüfung für den höheren Bibliotheksdienst ${ }^{20}$ bzw. für den gehobenen Dienst an Bibliotheken ${ }^{21}$ vom 2. Juli 2002 sowie durch die Verordnungen der NÖ Landesregierung über die Prüfung für den Fachdienst an Archiven, Bibliotheken und Museen ${ }^{22}$ bzw. für den fachlichen Hilfsdienst höherer Art an Archiven, Bibliotheken und Museen ${ }^{23}$ vom 16. Oktober 1973 geregelt. Nach der NÖ Ausbildungs- und Dienstprüfungsverordnung ${ }^{24}$ in der damaligen Fassung war für Bedienstete des höheren Bibliotheksdienstes und des gehobenen Dienstes an Bibliotheken vor dem Inkrafttreten der genannten Verordnungen der Besuch der vorgeschriebenen Ausbildungslehrgänge für Bundesbeamte vergleichbarer Verwendung und die Ablegung der für Bundesbeamte vorgesehenen Dienstprüfung vor der betreffenden Prüfungskommission des Bundes verpflichtend. ${ }^{25}$

Die interne Gliederung sieht die Referate (1) Bestandsaufbau und Erschließung ${ }^{26}$ mit den Unterbereichen (a) Druckschriftensammlung, (b) Sondersammlungen (Kartensammlung ${ }^{27}$ bzw. Topografische Sammlung ${ }^{28}$ ) und (c) Archivbibliothek und Zeitschriftenaufsatz-Bibliografie, ${ }^{29}$ (2) Erwerbung ${ }^{30}$ mit den Unterbereichen (a) Allgemeine Erwerbung ${ }^{31}$ und (b) Periodika ${ }^{32}$ und (3) Benutzung und Magazindienst ${ }^{33}$ mit den Unterbereichen (a) Benutzung und (b) Magazindienst vor. Daneben gibt es auf der Ebene der gesamten Abteilung Stabsstellen und Beauftragte wie Kanzlei und Kreditverwaltung, IT und Systemtechnik, Web und Öffentlichkeitsarbeit, Reproduktion und Restaurierung.

Die Niederösterreichische Landesbibliothek hat derzeit rund 20 Mitarbeiter. ${ }^{34}$ Jährlich stehen nach Abzug der von der Landesregierung einbehaltenen „Kreditsperre bei

20 LGBl. 2200/68-0.

21 LGBl. 2200/67-0.

22 LGBl. 2200/50-0.

23 LGBl. 2200/51-9.

24 LGBl. 2200/60-0.

25 Zugang zum Text all dieser Rechtsvorschriften ist über http://ww w.ris.bka.gv.at gegeben.

26 Bibliotheksdirektor und Referatsleiter: Mag. Hans-Joachim Alscher.

27 Kurator: Mag. Michael Duschanek.

28 Kurator: Mag. Dr. Ralph Andraschek-Holzer.

29 Kurator: Mag. Michael Bauer.

30 Referatsleiter: Mag. Herbert Schuster.

31 Leiterin: Elisabeth Fuchs.

32 Leiter: Andreas Geringer.

33 Referatsleiter: Mag. Dr. Achim Doppler.

34 Davon haben sechs einen Universitätsabschluss. 
Ermessensausgaben“ rund $200000 €$ für Anschaffungen und Betrieb zur Verfügung. ${ }^{35}$ Die Druckschriftensammlung umfasst einen Bestand von rund 380000 Bänden bei einem jährlichen Zuwachs von ca. 15000 Bänden und rund 4000 laufend gehaltene Zeitungen und Zeitschriften sowie rund 4000 Non-Book-Materialien. Dazu kommen über 50000 Dokumente aus der ehemaligen Burgenkundlichen Sammlung, ${ }^{36}$ rund 25000 Kartenblätter der Kartensammlung, über 25000 topografische Ansichten sowie über 60000 Ansichtskarten und Fotos der Topografischen Sammlung, rund 4500 Porträts, über 500 Flugschriften, rund 2500 Exlibris und rund 350 Kupferplatten, weiter rund 90000 Digitalisate von Sammelobjekten und 25000 sonstige Digitalisate. Die Katalogdatenbank umfasst neben der GND mit rund 11,5 Mio. Datensätzen und 18000 Form- bzw. Zeitschlagwörtern sowie über 8000 noch abzuarbeitenden lokalen Personen- und Schlagwortdatensätzen rund 520000 Titel $^{37}$ und rund 475000 ExemplarDatensätze. Über 45000 Datensätze wurden bisher in die GND eingebracht.

2014 haben knapp 9500 registrierte Benutzer über 6000 Medien außer Haus entlehnt und rund 500 digitale Reproduktionen von Medien erhalten. Fast 30 Leihgaben wurden allein für die Landesausstellung 2015 zur Verfügung gestellt. Bei rund 70 Veranstaltungen wurden rund 4000 Gäste empfangen. Nach Auskunft der Firma DABIS haben in diesem Jahr rund 10,8 Mio. Gesamtzugriffe auf den Onlinekatalog stattgefunden, ${ }^{38}$ im Einzelnen: 390000 Aufrufe der Startseite, 2,6 Mio. Listenanzeigen und 330000 Einzelanzeigen.

Das heutige, mit Oktober 1997 eröffnete und nach Plänen von Architekt Paul Katzberger aus Perchtoldsdorf von Architektin Karin Bily um umgerechnet 5,76 Mio.€ errichtete, mit hellen Steinen verkleidete, quaderförmige Bibliotheks-Hauptgebäude weist eine Gesamtnutzfläche von $5699 \mathrm{~m}^{239}$ auf. Unter Vernachlässigung des begehbaren, begrünten Flachdaches und der unter dem Gebäude befindlichen Tiefgarage besteht es aus drei Ebenen: Die Benutzungsebene im Obergeschoß umfasst, um den Lese$\mathrm{saal}^{40}$ angeordnet, einen Vortragsraum, die Topografische Sammlung, die Kartensammlung, den Zeitschriftensaal

35 Ohne die zentral getragenen Kosten für Gebäude, Personal und IT.

36 Ansichten, Zeitungsausschnitte, Pläne und Karten, Negative, Diapositive, Korrespondenzmappen.

37 Davon 400000 Druckschriften-Sammlung.

38 Davon 4,2 Mio. via Karlsruher Virtuellen Katalog (KVK).

39 Das entspricht einer Bruttogeschoßfläche von $6890 \mathrm{~m}^{2}$ und einer verbauten Fläche von $1428 \mathrm{~m}^{2}$ bei einer maximalen Höhe von 15,7 m. 40 Mit Infoschalter, Carrels, Computerstationen, Leseplätzen und systematisch aufgestelltem Freihandbestand. und das mit Festregalen ausgestattete Periodika-Magazin..$^{41} \mathrm{Im}$ Erdgeschoß befindet sich der Eingangsbereich (Foyer) samt WC-Anlagen und Garderoben mit Münzpfandschlössern, von dem aus ein Personenlift sowie die Haupttreppe in den Lesesaal führen. Hinter der Haupttreppe befindet sich eine weitere Treppe, von der man einerseits in den Ausstellungsraum samt vorgelagerter Loggia, andererseits in den Verwaltungsbereich gelangt, in dem auch das Niederösterreichische Volksliedarchiv ${ }^{42}$ sowie die Restaurier-Werkstätte untergebracht ist. Im Untergeschoß, aber immer noch auf Straßenniveau, befinden sich die beiden Hauptmagazine mit mechanisch betriebenen Rollregal-Anlagen und einer Stellfläche von rund 13000 Laufmetern. Die Buchaufstellung erfolgt hier nach dem Prinzip des Numerus currens mit Formattrennung. Erwähnenswert ist, dass das vom Architekten des Regierungsviertels Ernst Hoffmann ursprünglich vorgesehene Projekt eines gemeinsamen Gebäudes für Landesarchiv und Landesbibliothek als zu klein dimensioniert verworfen und der Komplex wie folgt vergeben wurde: Das Projekt von Architekt Paul Katzberger erhielt jeweils den Zuschlag für den Bau von Landesbibliothek sowie Landesarchiv. ${ }^{43}$ Die Bauausführung für die Landesbibliothek lag bei Architektin Karin Bily, für das Landesarchiv bei Architekt Michael Loudon. ${ }^{44}$ Als Architekt des 2009 fertiggestellten „Kulturdepots“ ${ }^{45}$ am Stadtrand fungierte Ernst Maurer.

\section{Wie es dazu kam, was vorher war: die diachrone Betrachtung}

Im Jahr 2013 beging die Niederösterreichische Landesbibliothek ihr zweihundertjähriges Jubiläum ${ }^{46}$ in Form der Ausstellung „Aufhebenswert“ ${ }^{47} \mathrm{zu}$ der ein umfangreicher Katalog mit dem von mir verfassten Beitrag „Geschichte

\section{Mit drei Ebenen.}

42 Träger: Volkskultur Niederösterreich GmbH mit Sitz in Atzenbrugg.

43 Das Landesarchiv liegt, durch einen Ahornhain getrennt, südseitig von der Landesbibliothek.

44 Weitere Gebäude des Kulturbezirks Sankt Pölten: Festspielhaus: Architekt Klaus Kada, Klangturm: Architekt Ernst Hoffmann, Landesmuseum nebst „Shedhalle“: Architekt Hans Hollein, ORF-Landesstudio: Architekt Gustav Peichl, NÖ Landesakademie = Haus Neue Herrengasse 17A: Architekt Ernst Hoffmann, (Tor zum Landhaus: Architekt Boris Podrecca).

45 Mit 7500 Laufmetern Stellfläche für die Landesbibliothek.

46 Gemeinsam mit dem hundertfünfzig Jahre alten Niederösterreichischen Landesarchiv.

47 Siehe www.aufhebenswert.at. 
der Niederösterreichischen Landesbibliothek“ und einer ausführlichen Bibliografie verwendeter und weiterführender Literatur erschienen ist. Um unnötige Wiederholungen zu vermeiden und dennoch das Wichtigste zur Geschichte unseres Hauses nicht unerwähnt zu lassen, versuche ich mich in einer gekürzten Wiedergabe des dort Gesagten:

Die Niederösterreichische Landesbibliothek geht auf die 1813 infolge eines entsprechenden Erlasses erfolgte Aufstellung der im gemeinsamen Besitz der niederösterreichischen Stände befindlichen Bücher im Prälatensaal des Niederösterreichischen Landhauses ${ }^{48}$ zurück, womit der Beginn der Ständischen Büchersammlung gelegt war. Vorerst verblieben die nicht im gemeinsamen Besitz befindlichen Bücher allerdings bei den einzelnen Ständen. ${ }^{49}$ So gelangte beispielsweise die auf einer Schenkung von Johann Joachim von Aichen (1664-1729) fußende Ritterstandsbibliothek erst 1823 in unseren Besitz. ${ }^{50}$ Erinnert sei an den Schriftsteller Ignaz Franz Castelli (1781-1862), der 1833 mit der Leitung der Bibliothek betraut wurde, wie auch an Alois König, der die Bibliothek von 1856-1895 vier Jahrzehnte lang prägte. Mit dem Ende der ständischen Verwaltung als Spätfolge des Revolutionsjahres 1848 wurde 1861 die neue Bezeichnung „Niederösterreichische Landesbibliothek“ offiziell. 1862 wurden Landesarchiv und Landesbibliothek aus der bisherigen Abhängigkeit von der Landschafts-Registratur entlassen. 1874 konnte erstmals ein gedruckter Bibliothekskatalog, der „Katalog der nieder-österr. Landesbibliothek in Wien“ ", ${ }^{51}$ vorgelegt werden. Einen weiteren Meilenstein bildete die Übernahme der Bibliothek des 1864 gegründeten Vereines für Landeskunde von Niederösterreich im Jahre 1886. Der Sekretär des Vereines Dr. Anton Mayer (1838-1924) wurde im Gegenzug zum Kustos für Bibliothek und Archiv bestellt und übte dieses Amt bis 1910 aus. In seine Wirkungszeit fällt daher auch die vom Landesausschuss 1896 erlassene Ordnung für das NÖ Landesarchiv und die NÖ Landesbibliothek. Als Leiter von Archiv und Bibliothek folgten ihm Dr. Max Vancsa (1866-1947) und Dr. Karl Lechner (1897-1975). ${ }^{52}$ Von 1938 bis 1948 leitete Dr. Kurt Vancsa (1904-1969), später Direktor der Bundesstaatlichen Studienbibliothek Linz,${ }^{53}$ die Geschicke der Niederösterreichischen Landesbibliothek.

48 In Wien I., Herrengasse 13.

49 Herrenstand, Ritterstand, Prälatenstand, freie Städte.

50 Die darin befindlichen Handschriften erhielt allerdings das Landesarchiv.

51 Mit Supplementen in den Jahren 1879 und 1891.

$52 \mathrm{Ab} 1923$.

53 Heute: Oberösterreichische Landesbibliothek.
Das 20. Jahrhundert war aus Sicht der Niederösterreichischen Landesbibliothek zunächst eine Geschichte von Bestandsverlusten: So mussten 1922 nach der Erhebung Wiens zum selbständigen Bundesland und infolge der Schaffung von Groß-Wien 1943 Bestände an das Land Wien abgetreten werden. ${ }^{54}$ Nach dem Krieg wurde verbotene Literatur nationalsozialistischer Autoren abgestoßen und an die Universitätsbibliothek Wien abgetreten. Nutznießer der Abtretungen an das Land Wien war das WienMuseum Karlsplatz ${ }^{55}$ und nicht die Wienbibliothek im Rathaus. ${ }^{56}$ In die Ära von Dr. Josef König (1903-1982), der der Landesbibliothek von 1948 bis 1969 vorstand, fällt der erfolgreiche Kampf um den Erhalt von Kartensammlung und Topografischer Sammlung, die gemäß Beschluss der Landesregierung vom 27. September 1966 an das Niederösterreichische Landesmuseum abgetreten hätten werden sollen. Aus ihrer ursprünglichen Heimstätte, dem seit 1513 als solches fungierenden Niederösterreichischen Landhaus, ${ }^{57}$ wurde die Landesbibliothek 1967 aus Platzgründen an den neuen Standort in der Teinfaltstraße 8 in Wien I. übersiedelt. ${ }^{58}$ Seit der Übersiedlung der Landesbibliothek in die neue Hauptstadt Sankt Pölten dient dieser Standort als (zunächst provisorisch gedachte) Außenstelle der Universitätsbibliothek Wien.

Infolge des durch die Übersiedlung ausgelösten Modernisierungsschubes wurde 1969 die Restaurier-Werkstätte ${ }^{59}$ in Betrieb genommen, zugleich erfolgte der Umstieg vom herkömmlichen Bandkatalog auf einen nach „Preußischen Instruktionen“ geführten Zettelkatalog. Die Altkataloge wurden bis 1988 auf Zettelkatalogform umgeschrieben. Einmal auf den Geschmack gekommen, war der Fortschrittswille nicht mehr aufzuhalten: Bereits 1984 wurde ein Online-Katalog ${ }^{60}$ in Betrieb genommen. Als Volltext-Retrieval-System diente „STAIRS“ von IBM. Die Katalogisierer vermerkten vorzunehmende Korrekturen

54 Die kriegsbedingte Auslagerung der Bestände in das Kloster Zwettl hat hingegen keine bekannten Verluste nach sich gezogen. 55 Früher: Historisches Museum der Stadt Wien.

56 Früher: Wiener Stadt- und Landesbibliothek.

57 Jetzt „Palais Niederösterreich“, Ausgangspunkt der Revolution von 1848 und Gründungsort der Republik Österreich.

58 Ursprünglich Bankgebäude der 1929 infolge der Weltwirtschaftskrise in Konkurs geschlitterten Bodencreditanstalt und zuletzt Sitz der niederösterreichischen Energieversorger NEWAG und NIOGAS (heute: EVN).

$59 \mathrm{Zu}$ deren Prunkstücken der Prototyp eines PapieranfaserungsGerätes von 1970, eigentlich ein umgebauter Blattbildner, gehört. 60 Genauer gesagt: ein eigens entwickeltes Einplatz-Erfassungssystem, dessen Daten in regelmäßigen Abständen mittels Floppy Disk auf einen IBM-Großrechner transferiert wurden, der von zahlreichen Landesdienststellen via Terminal erreichbar war. 
handschriftlich auf Ausdrucken der Katalogisate. Mangels OPAC (Online Public Access Catalogue) wurden Katalogkarten nach „Preußischen Instruktionen“ für den weitergeführten Publikumskatalog wie Beamtenkatalog ausgedruckt, obwohl die Formalerschließung bereits (etwas halbherzig) nach den Regeln für die alphabetische Katalogisierung in wissenschaftlichen Bibliotheken (RAK-WB) erfolgte. Der Altbestand (Band- bzw. Zettelkatalog) wurde in einer eigenen Datenbank durch Abtippen zur Gänze nacherfasst. Mit dem Jahreswechsel 1993/1994 erfolgte der Umstieg auf das Bibliothekssystem BIS-C bei gleichzeitiger Einstellung des Zettelkatalogs und konsequenter Anwendung der Regeln für die alphabetische Katalogisierung in wissenschaftlichen Bibliotheken (RAK-WB) und der Regeln für den Schlagwortkatalog (RSWK) sowie der als MAB-Diskettendienst bezogenen Normdateien. ${ }^{61}$ Die in eine eigene Datenbank konvertierten Altbestände wurden 1997 integriert. 1998 erfolgte die Inbetriebnahme des WebOPACs (Online Public Access Catalogue), der 1999 die Schaffung des virtuellen Verbundes der Landesbibliotheken Österreich und Südtirol und 2003 dessen Integration in den Karlsruher Virtuellen Katalog (KVK) sowie 2004 der Start des Österreichischen Normdatenverbundes (ÖNDV) als eigenständige Redaktion (Clearingstelle) für die Einbringung von Normdaten folgte. Nach Integration der weiteren Datenbanken (wie Kartensammlung, Topografische Sammlung etc.) im Jahre 2012 und Implementierung der Gemeinsamen Normdatei (GND) im Jahre 2013 sehen wir dem augenblicklichen Umstieg auf Resource Description and Access (RDA) trotz aller dafür erforderlichen Anstrengungen vergleichsweise gelassen entgegen ...

Dr. Josef König war als Bibliotheksdirektor 1969 von Dr. Rudolf Kreitmayr und dieser $1978^{62}$ von Dr. Eduard Ronge beerbt worden. Ihm folgte 1989 Dr. Hermann Riepl und 1996, unmittelbar vor der Übersiedlung nach Sankt Pölten, Dr. Gebhard König. ${ }^{63}$ Bereits 1984 war die Niederösterreichische Landesbibliothek eine eigenständige Abteilung des Amtes der NÖ Landesregierung ${ }^{64}$ geworden, verlor diesen Status aber 2011 nach der Pensionierung von Dr. Gebhard König wieder durch Zusammenlegung mit dem Niederösterreichischen Landesarchiv. Seit damals fungiere ich als Bibliotheksdirektor (und Stellvertreter des

61 Schlagwortnormdatei (SWD) ab 1995, in der Folge auch Gemeinsame Körperschaftsdatei (GKD) und Personennamendatei (PND).

62 De facto schon 1976.

63 Seit 2000 außerdem Leiter der Gruppe Kultur, Wissenschaft und Unterricht des Amtes der NÖ Landesregierung.

64 III/4, später K3.
Abteilungsleiters Archivdirektor Dr. Willibald Rosner). Besonders erwähnenswerte Meilensteine waren das bereits erwähnte Inkrafttreten des Mediengesetzes am 1. Januar 1982, ${ }^{65}$ die von einer Festschrift begleitete Sonderausstellung „175 Jahre Niederösterreichische Landesbibliothek“ im Jahre 1988 und die Eröffnung des Neubaus in Sankt Pölten am 1. Oktober 1997, ${ }^{66}$ der 25. Österreichische Bibliothekartag 1998 in Sankt Pölten ${ }^{67}$ sowie in gewisser Weise auch die Messe von Papst Johannes Paul II. am 20. Juni 1998 auf dem damals noch nicht verbauten Platz vor der nördlichen Hauswand des neuen Bibliotheksgebäudes. An bedeutenden Publikationen der Landesbibliothek aus jüngerer Zeit sei vor allem das 2005 bis 2010 im Verlag Christian Brandstätter erschienene, sechsbändige Werk „Niederösterreich in alten Ansichten" genannt.

\section{Was man nicht verschweigt, aber am besten zeigt}

Bilder sagen mehr als Worte: Die Landesbibliothek und ihre Sammlungen seien abschließend durch Abbildungen einiger ausgewählter Objekte repräsentiert.

Dabei handelt es sich um Abbildungen des ältesten gedruckten Buches aus Niederösterreich, um einen Stammbaum und eine wertvolle Handschrift vom Ende des 15. Jahrhunderts sowie um das wahrscheinlich älteste Industrie-und-Glück-Tarock aus dem frühen 19. Jahrhundert. Schlussendlich runden die Kupferstiche des Kartografen Georg Matthäus Vischer vom Ende des 17. Jahrhunderts nebst einer dazugehörigen Kupferplatte die Beschreibung des Bestands der Niederösterreichischen Landesbibliothek ab.

65 Das uns zu einer Pflichtexemplar-Bibliothek machte. 66 Ebenfalls mit einer Festschrift.

67 Der Österreichische Bibliothekartag wird im Zweijahresrhythmus von der Vereinigung Österreichischer Bibliothekarinnen und Bibliothekare (VÖB) veranstaltet, die im Gegensatz zum institutionell organisierten Büchereiverband Österreichs (BVÖ) als berufliche Interessensvertretung von in wissenschaftlichen Bibliotheken, Dokumentations- und Informationseinrichtungen tätigen Personen anzusehen ist. 


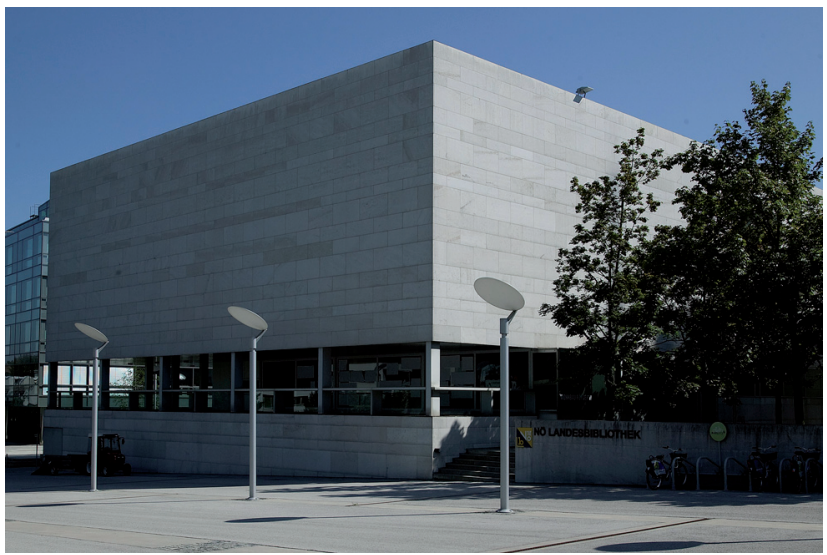

Abb.1: Außenansicht des Bibliotheksgebäudes (von Westen)

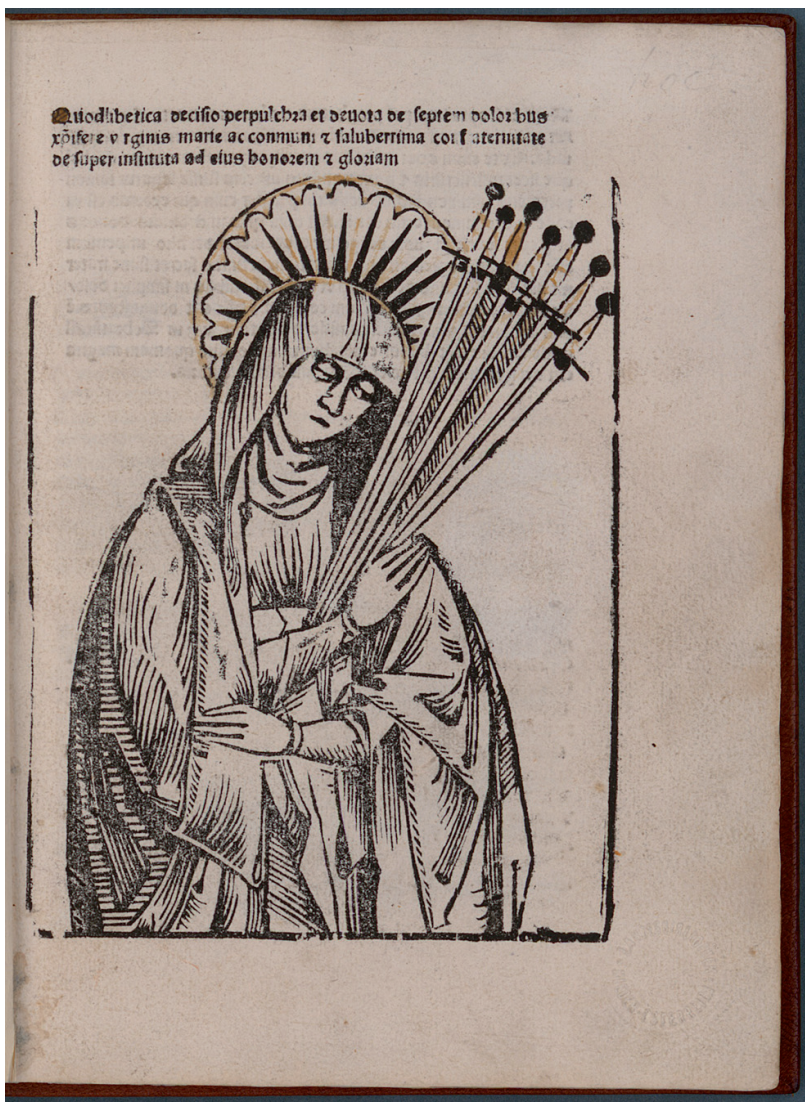

Abb. 3: François, Michel: Quodlibetica decisio perpulchra et devota de septem doloribus christifere virginis Mariae ac communi et saluberrima confraternitate de super instituta ad eius honorem et gloriam. Schrattenthal 1501. Signatur 35.176 B. Titelblatt (Ältestes gedrucktes Buch aus Niederösterreich)

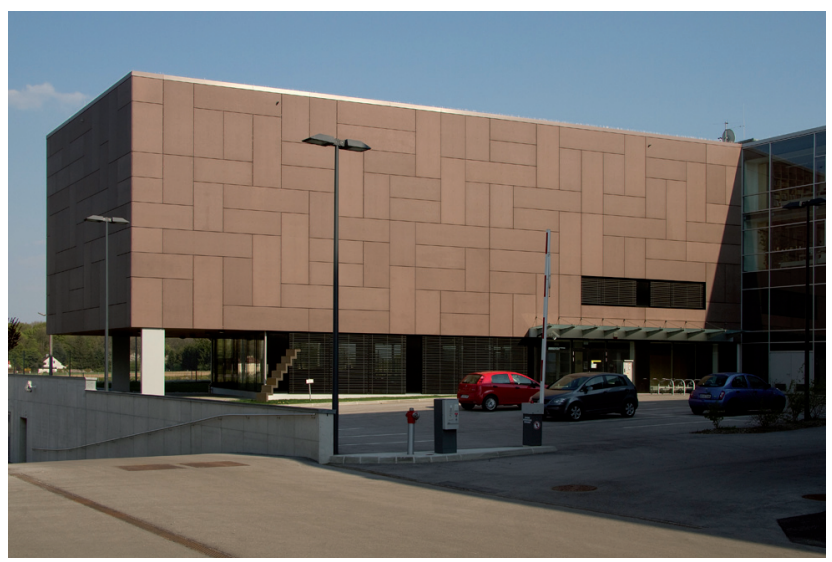

Abb. 2: Außenansicht des „Kulturdepots“, Archiv- und Bibliothekstrakt

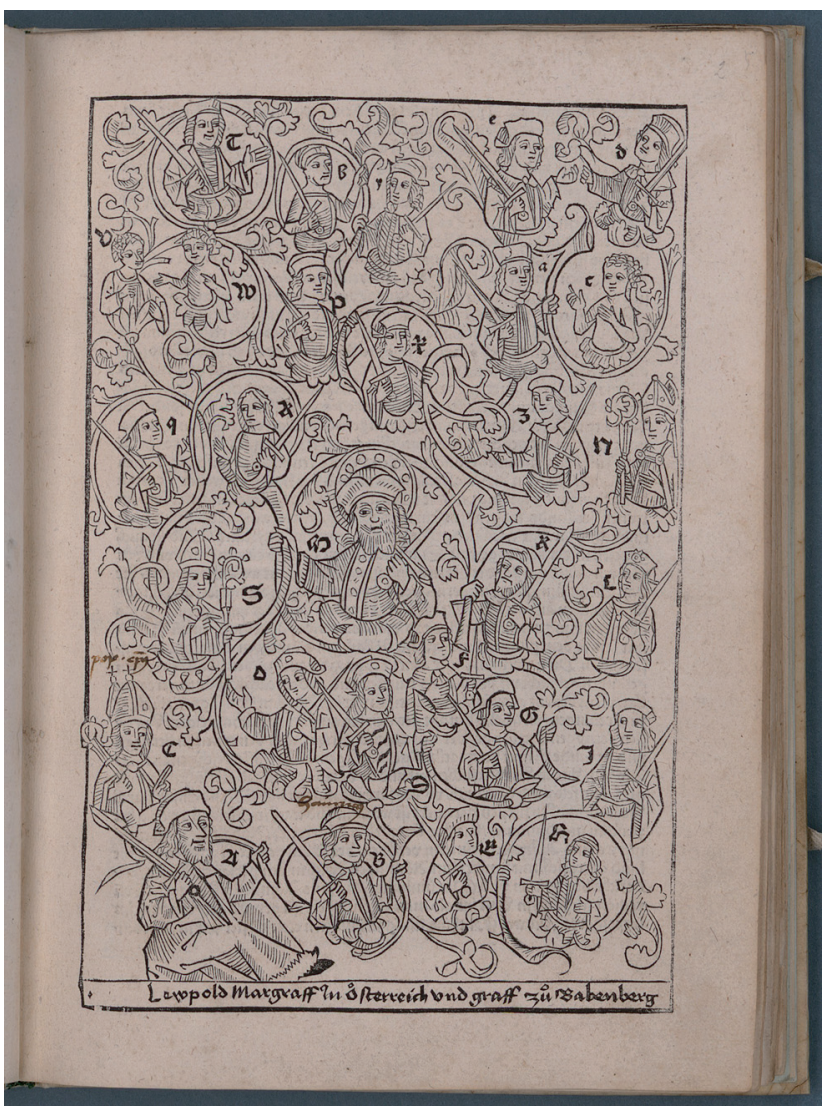

Abb. 3a: Suntheim, Ladislaus: Der löblichen Fürsten und des Landes Österreich Altherkommen und Regierung. Basel 1491. Signatur 3.571 C. Babenberger-Stammbaum (vgl. Triptychon von Hans Part im Stift Klosterneuburg) 

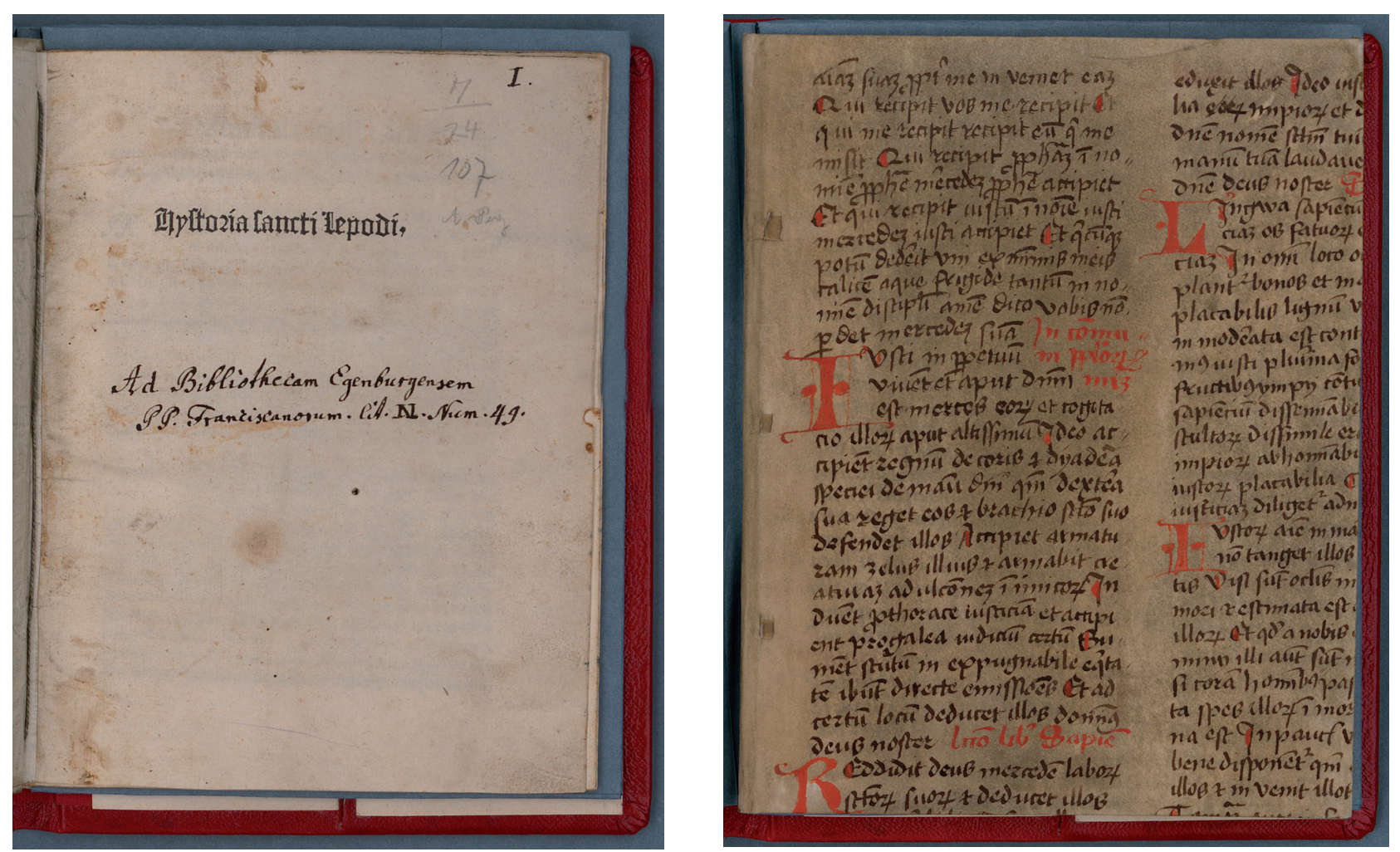

Abb. 4: Historia Sancti Leopoldi. Passau, ca. 1489. Signatur 139.392 B. Titelseite (links) und Einband (rechts) ${ }^{68}$

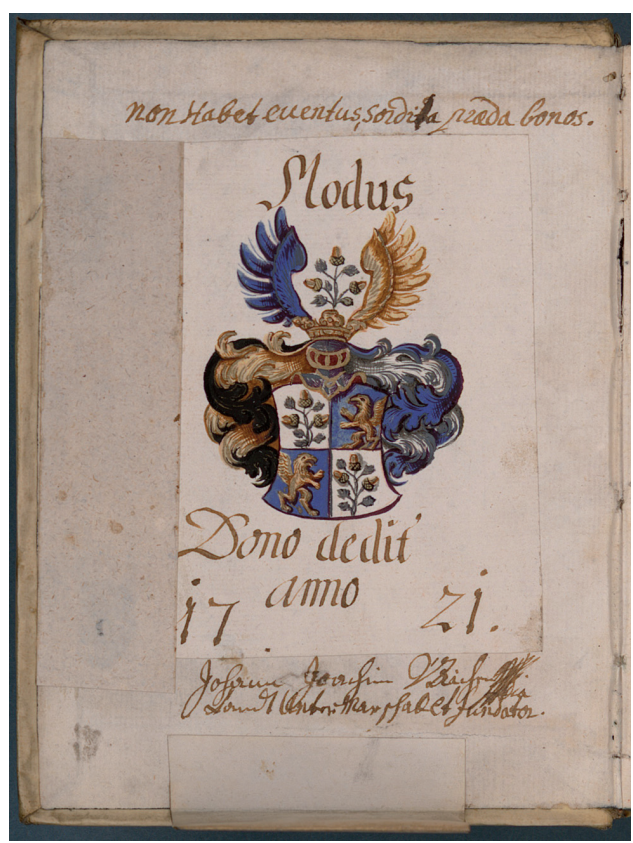

Abb. 5: Wappenexlibris des Johann Joachim von Aichen (1664-1729) mit Widmung aus dem Jahre 1721

68 Diese Inkunabel steht wie die zuvor gezeigte (von Ladislaus Suntheim) in Zusammenhang mit der 1485 erfolgten Heiligsprechung des Babenbergischen Markgrafen Leopold III., seit 1663 Landespatron von Niederösterreich.

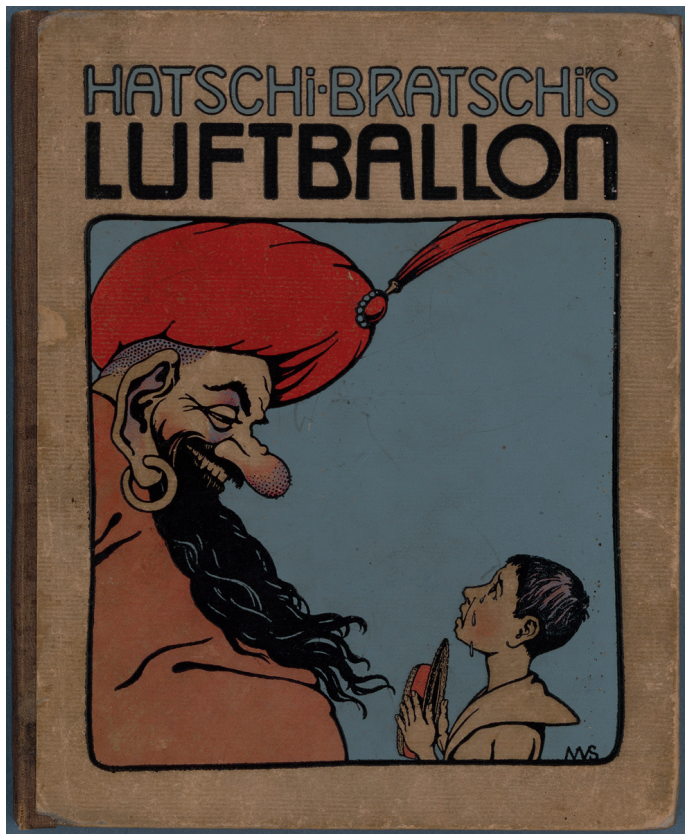

Abb. 6: Ginzkey, Franz Karl: Hatschi-Bratschi’s Luftballon. Bilder von M. von Sunnegg. Berlin 1904. Signatur 135.417 B. Cover der Erstausgabe $^{69}$

69 Von Franz Karl Ginzkey stammt auch der Text der heutigen Niederösterreichischen Landeshymne. 


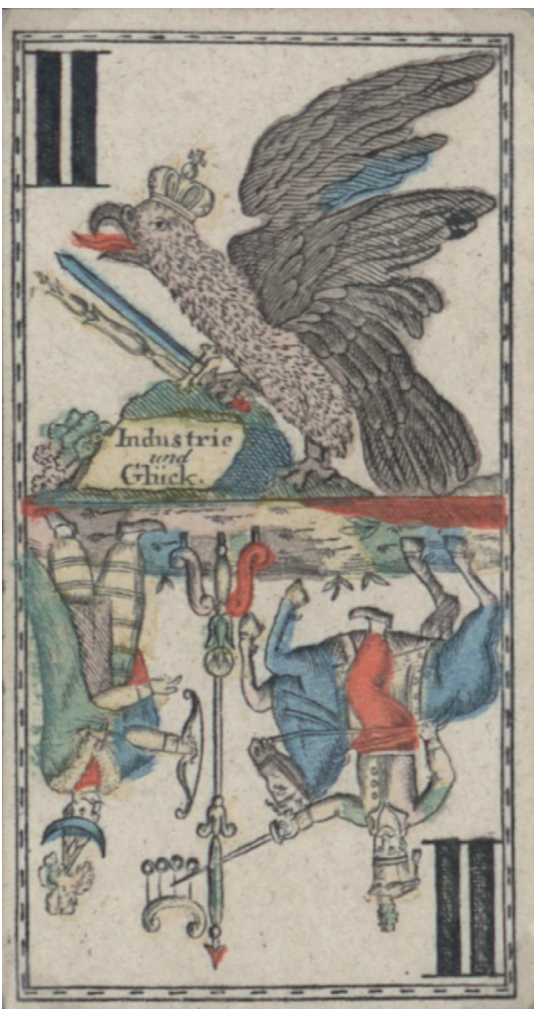

Abb. 7: Industrie-und-Glück-Tarock von Johann Norbert Hofmann, Wien 1815. Signatur AV VIII 10. Tarock II (Das bisher älteste bekannte Industrie-und-Glück-Tarock. Dazu Faksimile-Ausgabe von Piatnik 2013)

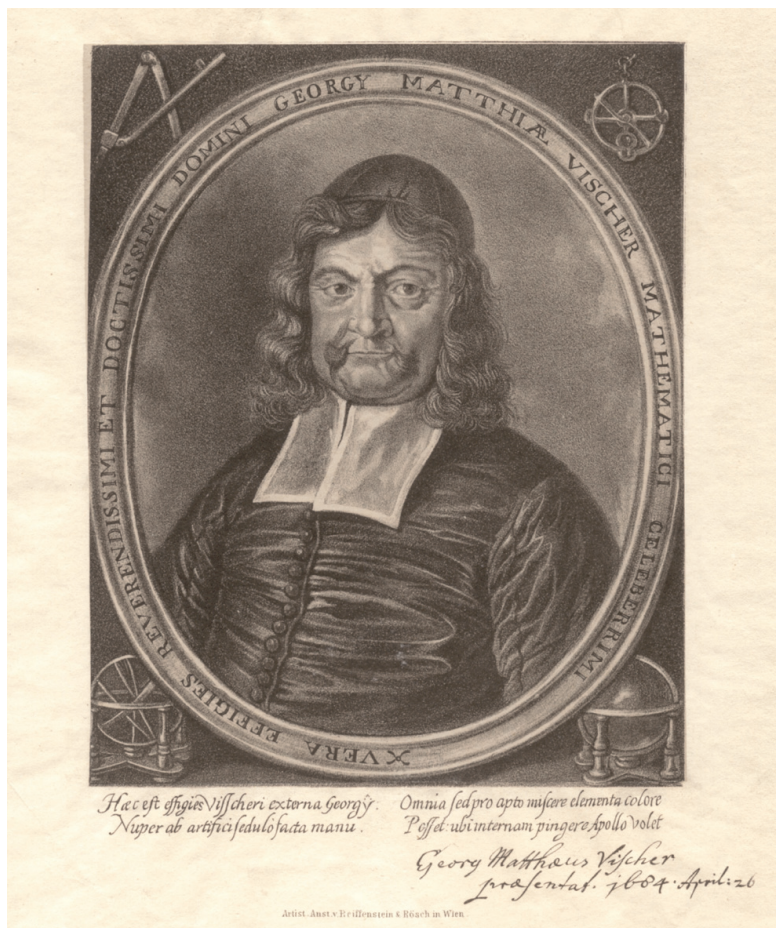

Abb. 9: Porträt von Georg Matthäus Vischer, Lichtdruck nach Kupferstich von 1684, Signatur P 2.089

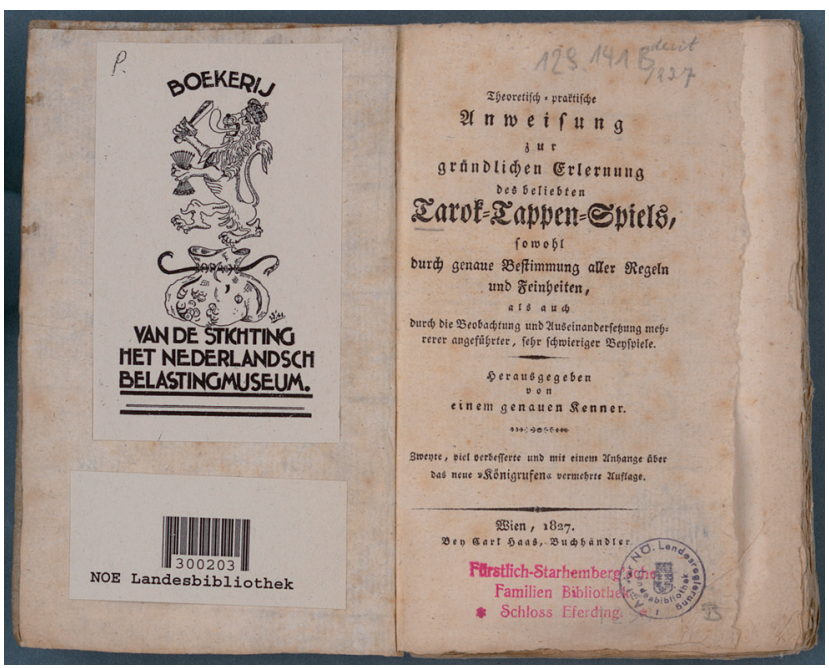

Abb. 8: Theoretisch-praktische Anweisung zur gründlichen Erlernung des beliebten Tarok-Tappen-Spiels. Zweite Auflage. Wien 1827. Signatur 129.141 B (Enthält die älteste Beschreibung des Königrufens)

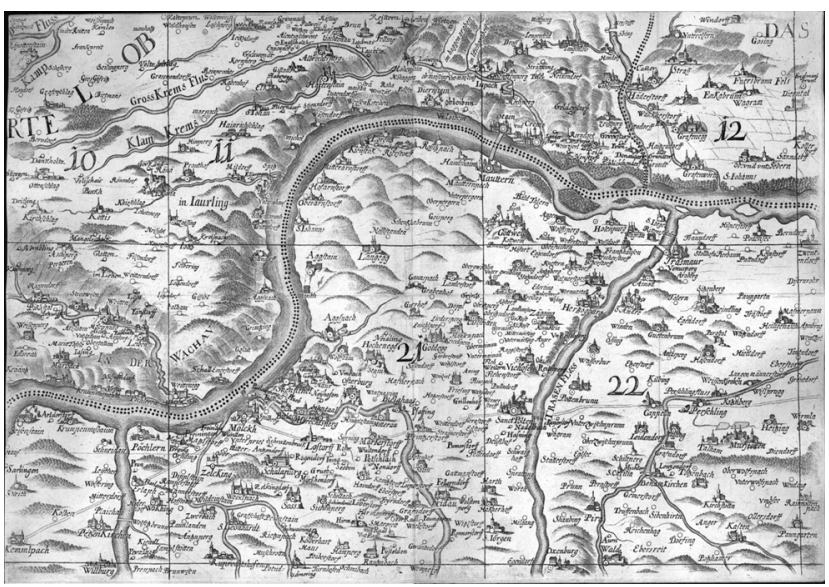

Abb. 10: Vischer, Georg Matthäus: Archiducatus Austriae Inferioris geographica et noviter emendata accuratissima descriptio. Signatur AV 227 / 169. Blatt 6 (Raum Sankt Pölten) ${ }^{70}$

70 Vom Kartografen Georg Matthäus Vischer (1628-1696) wurde die im Auftrag der Stände angefertigte Niederösterreichkarte von 1670 erstellt (dem oben gezeigten Exemplar liegt die von Jacob Hoffmann und Jakob Hermundt gestochene Ausgabe von 1697 zugrunde). Als „Nebenprodukt“ wurden Doppelansichten wie die vorliegende gestochen. Bemerkenswert ist, dass wir zu den niederösterreichischen Stichen auch die zugrundeliegenden Kupferplatten besitzen. Außer für Niederösterreich fertigte Vischer in gleicher Weise Stiche für Oberösterreich und die Steiermark an. 


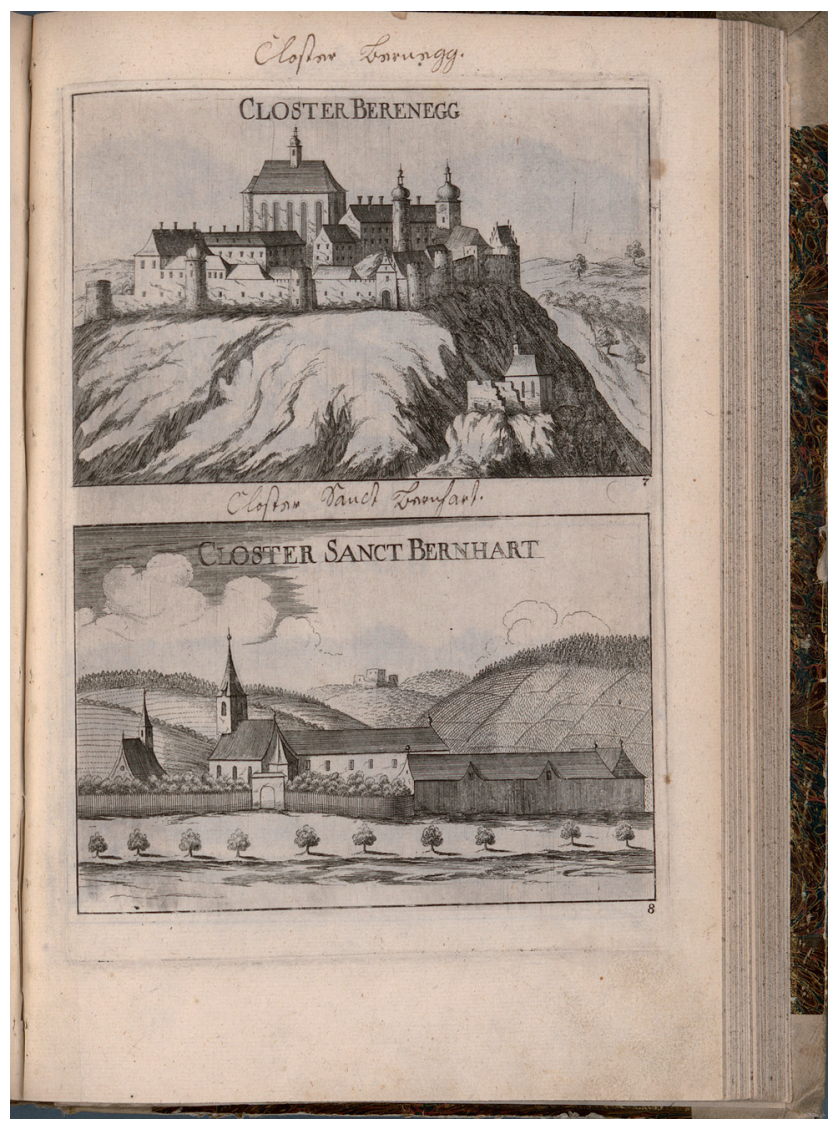

Abb. 11: Vischer, Georg Matthäus: Topographia archiducatus Austriae Inferioris modernae. 1672. Signatur 760 C. Blatt Closter Berenegg [Stift Pernegg] / Closter Sankt Bernhart [Kloster St. Bernhard]

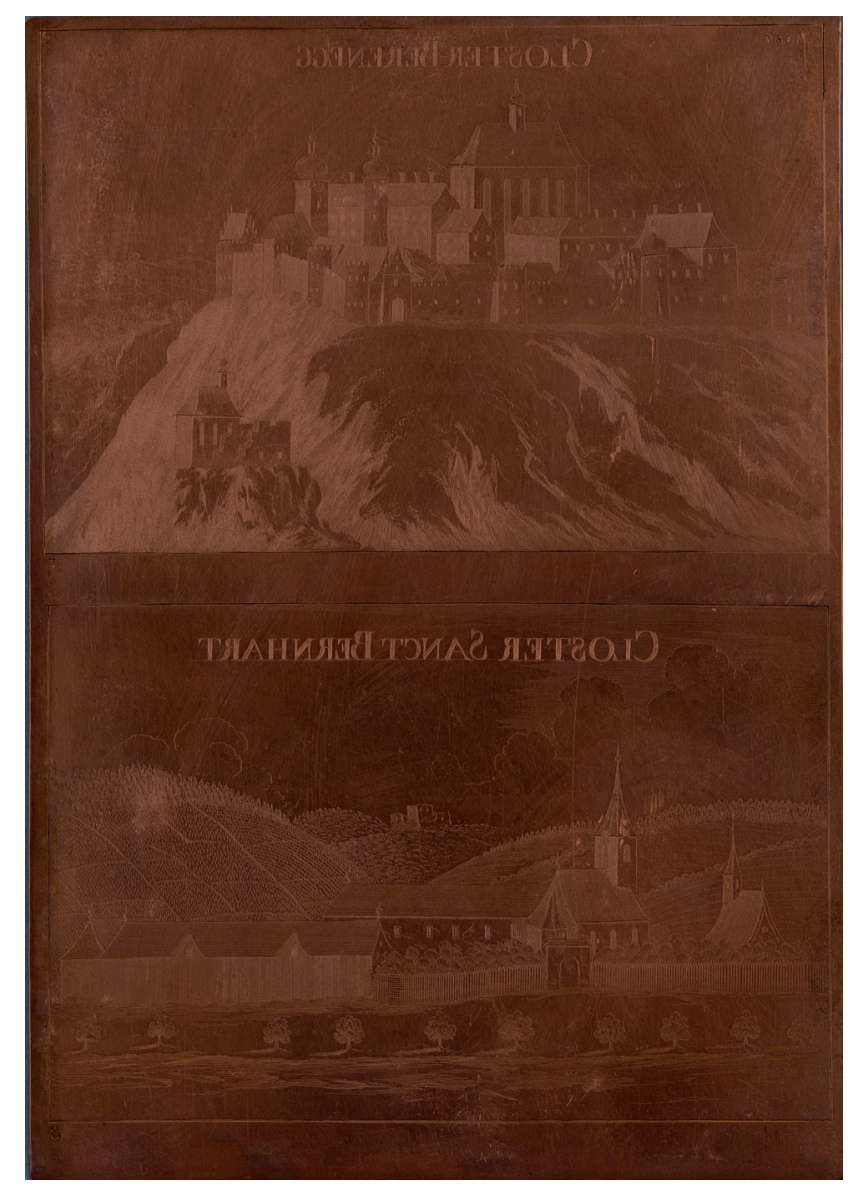

Abb. 12: Kupferplatte dazu (Nr. 187 V. O. M. B.)

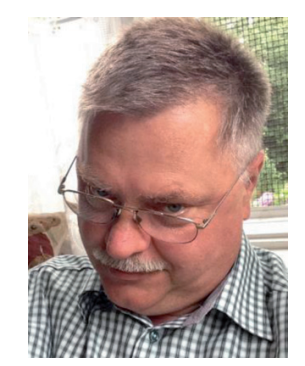

wHR Mag. Hans-Joachim Alscher Bibliotheksdirektor

Stv. Leiter der Abt. NÖ Landesarchiv und NÖ Landesbibliothek A-3109 Sankt Pölten Österreich hans.alscher@noel.gv.at 